\title{
BAND-GAP RENORMALIZATION IN \\ QUASI-ONE-DIMENSIONAL SYSTEMS
}

\author{
B. TANATAR \\ Department of Physics, \\ Bilkent University, \\ 06533, Ankara, Turkey
}

\section{Introduction}

A dense electron-hole plasma forming in a semiconductor under intense laser excitation comprises an interesting many-body system. Because of the exchange effects and the screening of the Coulomb interaction, the single-particle properties are renormalized. A notable phenomenon is the band-gap renormalization as a function of the plasma density which is important to determine the emission wavelength of coherent emitters as being used in semiconductors.[1] As a substantial amount of carrier population may be induced by optical excitation, the renormalized band gap can affect the excitation process in turn and lead to optical nonlinearities. In this paper we investigate the density dependence of the band-gap renormalization (BGR) in quasi-one-dimensional (Q1D) photoexcited semiconductors. Under high optical excitation the band gap for 2D and bulk systems is found to decrease with increasing plasma density due to exchange-correlation effects. The observed band gaps are typically renormalized by $\sim 20 \mathrm{meV}$ within the range of plasma densities of interest which arise chiefly from the conduction band electrons and valence band holes. In the Q1D structures based on the confinement of electrons and holes, the electron-hole plasma is quantized in two transverse directions, thus the charge carriers essentially move only in the longitudinal direction. Recent progress in the fabrication techniques such as molecular-beam epitaxy (MBE) and lithographic deposition have made possible the realization of such quasi-one-dimensional systems.[2] Band-gap renormalization as well as various optical properties of the Q1D electron-hole systems have been studied[3, 4, 5, 6, 7] similar to 
the bulk (3D) and quantum-well (2D) semiconductors[8, 9, 10, 11] where generally good agreement with the corresponding measurements[12] exist.

Our main motivation comes from the recent experiments of Cingolani et al. $[13,14]$ in which they investigated the carrier density dependence of a quasi-one-dimensional electron-hole plasma confined in GaAs quantum wires using luminescence spectra. Comparing the band-gap data with the available calculations, Cingolani et al.[13] pointed out the need for a more realistic calculation. Density dependence of the BGR in Q1D systems was first considered by Benner and Haug[3] within the quasi-static approximation as previously employed for $2 \mathrm{D}$ and $3 \mathrm{D}$ systems.[8, 9, 10, 11] In a detailed study that appeared recently $\mathrm{Hu}$ and Das Sarma[4] also calculated the BGR, neglecting the hole population and considering an electron plasma confined in the lowest conduction subband only. The results of $\mathrm{Hu}$ and Das Sarma[4] are rather close to the experimental data.[13]

In this study our aim is to calculate the BGR using a statically screened approximation which is based on the RPA. We employ the temperature dependent, static, RPA dielectric function and address the question of validity of using the plasmon-pole approximation to it. We investigate the temperature dependence of the BGR at various electron-hole plasma densities and quantum well widths. We also discuss the effects of electron-phonon interactions.

The rest of this paper is organized as follows. In the next section we give a brief outline of the static screening approximation (quasi-static approximation). In Section III we present our results for the BGR in Q1D electron-hole plasmas and compare them with the experiments. Finally, we conclude with a brief summary of our main results.

\section{Theory}

For the Q1D system we consider a square-well of width $a$ with infinite barriers. It may be built from a Q2D quantum-well (grown in the $z$-direction) by introducing an additional lateral confinement. We assume that effective mass approximation holds and for GaAs take $m_{e}=0.067 m$, and $m_{h}=0.2 m$, where $m$ is the bare electron mass. Note that we have chosen the hole effective mass to reproduce on average the dispersion of the four topmost 1D subbands to conform with the experimental analysis of Ref. 13 . The effective Coulomb interaction between the charge carriers is given by[4]

$$
V(q)=\frac{2 e^{2}}{\epsilon_{0}} \int_{0}^{1} d x K_{0}(q a x)\left[(1-x)[2+\cos (2 \pi x)]+\frac{3}{2 \pi} \sin (2 \pi x)\right],
$$

in which $K_{0}(x)$ is the zeroth-order modified Bessel function of the second kind, and $\epsilon_{0}$ is the lattice dielectric constant. Due to the presence of 
an electron-hole plasma, assumed to be in equilibrium, the bare Coulomb interaction is screened. The equilibrium assumption is justified since the laser pulse durations are typically much longer than the relaxation times of the semiconductor structures under study. Defining the statically screened Coulomb interaction as $V_{s}(q)=V(q) / \varepsilon(q)$, we consider the dielectric function in the random-phase-approximation (RPA)

$$
\varepsilon(q)=1-2 V(q) \sum_{i, k} \frac{f_{i}(k)-f_{i}(k+q)}{\epsilon_{i}(k)-\epsilon_{i}(k+q)+i \eta}
$$

where the index $i=e, h$, and $\epsilon_{i}(k)=\hbar^{2} k^{2} / 2 m_{i}$ are the bare single-particle energies. In most previous studies, the dielectric function $\varepsilon(q)$ was further simplified by the plasmon-pole approximation. Here we use the full static RPA at finite temperature without resorting to any approximations and discuss in the following section the validity of the plasmon-pole approximation.

Assuming a homogeneously distributed electron-hole plasma in thermal equilibrium the electron and hole distribution functions are written as

$$
f_{i}(k)=\frac{1}{e^{\beta\left(\epsilon_{i}(k)-\mu_{i}^{0}\right)}+1},
$$

where $\beta=1 / k_{B} T$ and $\mu_{i}^{0}$ are the inverse carrier temperature and (unrenormalized) chemical potential of the different species, respectively. The plasma density $N$ determines $\mu_{i}^{0}$ through the normalization condition $N=$ $2 \sum_{k} f_{i}(k)$.

Adopting the quasi-static approximation $[8,9]$ which amounts to neglecting the recoil effects relative to the plasma frequency in the full frequency dependent expressions, we decompose[8, 9] the electron and hole self-energies into screened exchange (sx) and Coulomb hole (Ch) terms: $\Sigma_{i}(k)=\Sigma_{i}^{\mathrm{sx}}(k)+\Sigma_{i}^{\mathrm{Ch}}$, where

$$
\Sigma_{i}^{\mathrm{sx}}(k)=-\sum_{k^{\prime}} V_{s}\left(k-k^{\prime}\right) f_{i}\left(k^{\prime}\right), \quad \text { and } \quad \Sigma_{i}^{\mathrm{Ch}}=\frac{1}{2} \sum_{k^{\prime}}\left[V_{s}\left(k^{\prime}\right)-V\left(k^{\prime}\right)\right] .
$$

The above set of equations may be derived[8] from the dynamical self-energy expressions by neglecting all recoil energies with respect to the plasma frequency. As in the case of $2 \mathrm{D}$ and $3 \mathrm{D}$ calculations $[8,9,10,11]$ we assume that the BGR results from rigid bandshifts; i.e., the self-energies depend only weakly on wave vector $k$. The band-gap renormalization is then given by

$$
\Delta E_{g}=E_{g}^{\prime}-E_{g}=\Sigma_{e}(0)+\Sigma_{h}(0),
$$


namely the electron and hole self-energies calculated at the respective band edges. Within the same spirit, we calculate the renormalized total chemical potential of the electron-hole plasma using

$$
\mu_{T}=\sum_{i}\left[\mu_{i}^{0}+\Sigma_{i}\left(k_{F}\right)\right]
$$

in which $k_{F}=\pi N / 2$ is the Fermi wave vector. The self-energy part in the above expression is also called the exchange-correlation contribution $\mu_{\mathbf{x c}}$ to the chemical potential.

\section{Results and discussions}

In Fig. 1 we show the results of our calculation for the BGR (indicated by the solid curve) as a function of the electron-hole plasma density $N$. In order to make a ready comparison with the experimental results of Cingolani et al.[13] (shown by full circles), we have evaluated $\Delta E_{g}$ for a quantum wire of width $a=500 \AA$, at $T=100 \mathrm{~K}$. The investigated[13] quantum wires were fabricated by plasma etching from quantum-well structures with lateral widths of $600 \pm 50 \AA$. The experimental data have been collected over a whole set of spectra at various carrier temperatures. As we shall demonstrate below the BGR is not very sensitive to the temperature and we obtain rather good agreement with the experimental results. The zero-temperature calculation of $\mathrm{Hu}$ and Das Sarma[4] also represents well the Cingolani et al.[13] data, rendering the insensitivity of $\Delta E_{g}$ to temperature in the range of densities reported. The agreement between our calculated results and the experiment appears to be rather good. However, we caution that the experimental data points[13] were extracted from the observed luminescence spectra by assuming a free-carrier model. Our calculations indicate the importance of Coulomb effects. Thus a more refined line-shape analysis would be required to render the comparison more meaningful. To assess the relative importance of the various contributions to $\Delta E_{g}$ we display in Fig. 2 the screened-exchange (dashed line) and Coulomb hole (dotted line) parts of the self-energy. It is observed that the Ch term dominates for low densities $\left(N \leq 3 \times 10^{5} \mathrm{~cm}^{-1}\right)$ but the total $\Delta E_{g}$ is mainly determined by the sx term at high densities. This situation is somewhat different than the case in $2 \mathrm{D}$ systems, where the $\mathrm{Ch}$ term approximates the band-gap renormalization satisfactorily for the relevant density regime.[15] On the other hand, a recent work[6] on InGaAs/InP quantum wires made use of the unscreened exchange energy (Hartree-Fock) only to account for the subband renormalizations.

The dotted curve in Fig. 1 gives the BGR calculated within the plasmonpole approximation to the dielectric function using the same parameters. In 


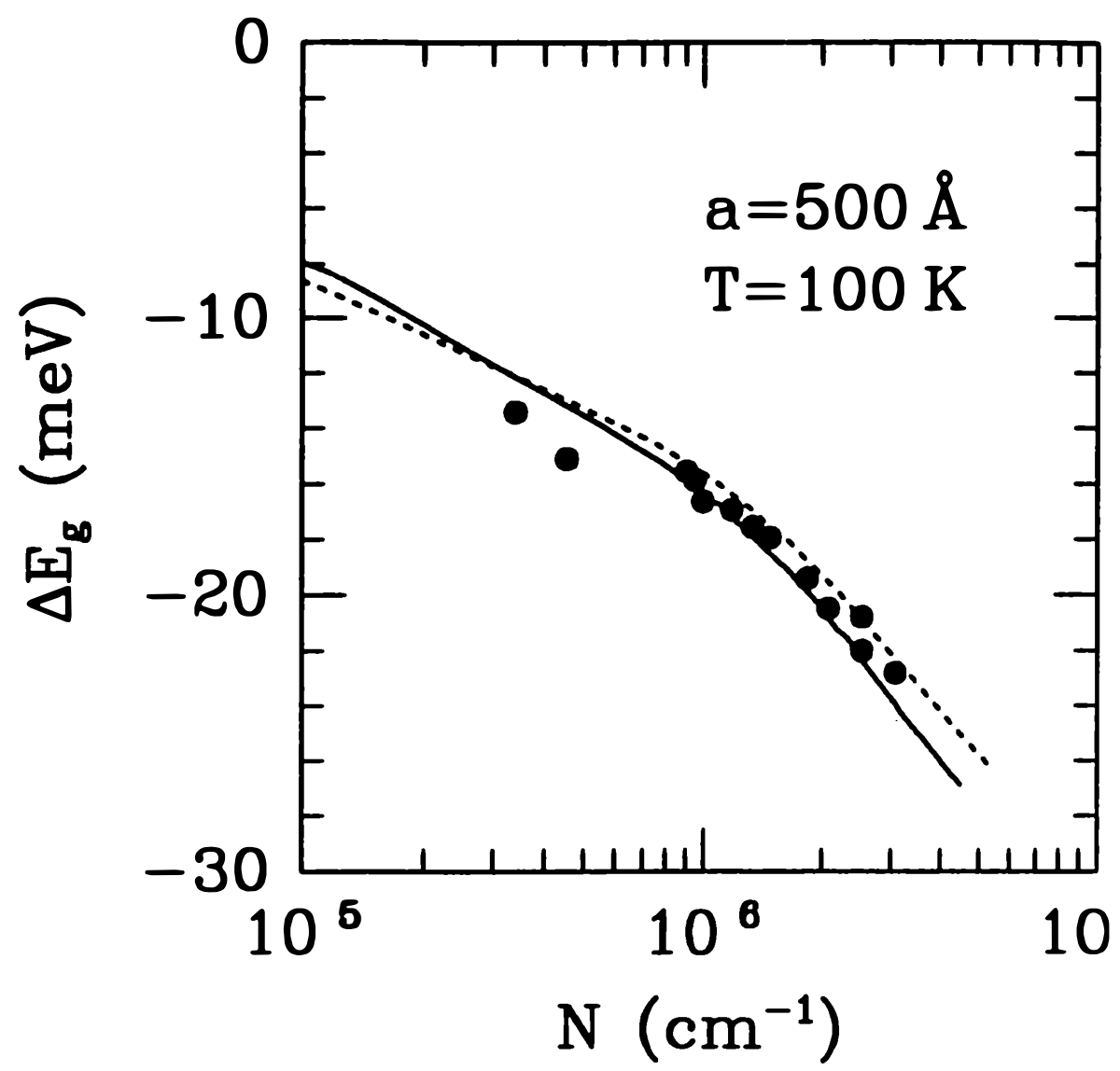

Figure 1. The calculated band-gap renormalization $\Delta E_{g}$ as a function of the electron-hole pair density. Full circles are the experimental results from Ref.13. Solid and dotted lines are calculated with the full RPA dielectric function and the plasmon-pole approximation to it, respectively.

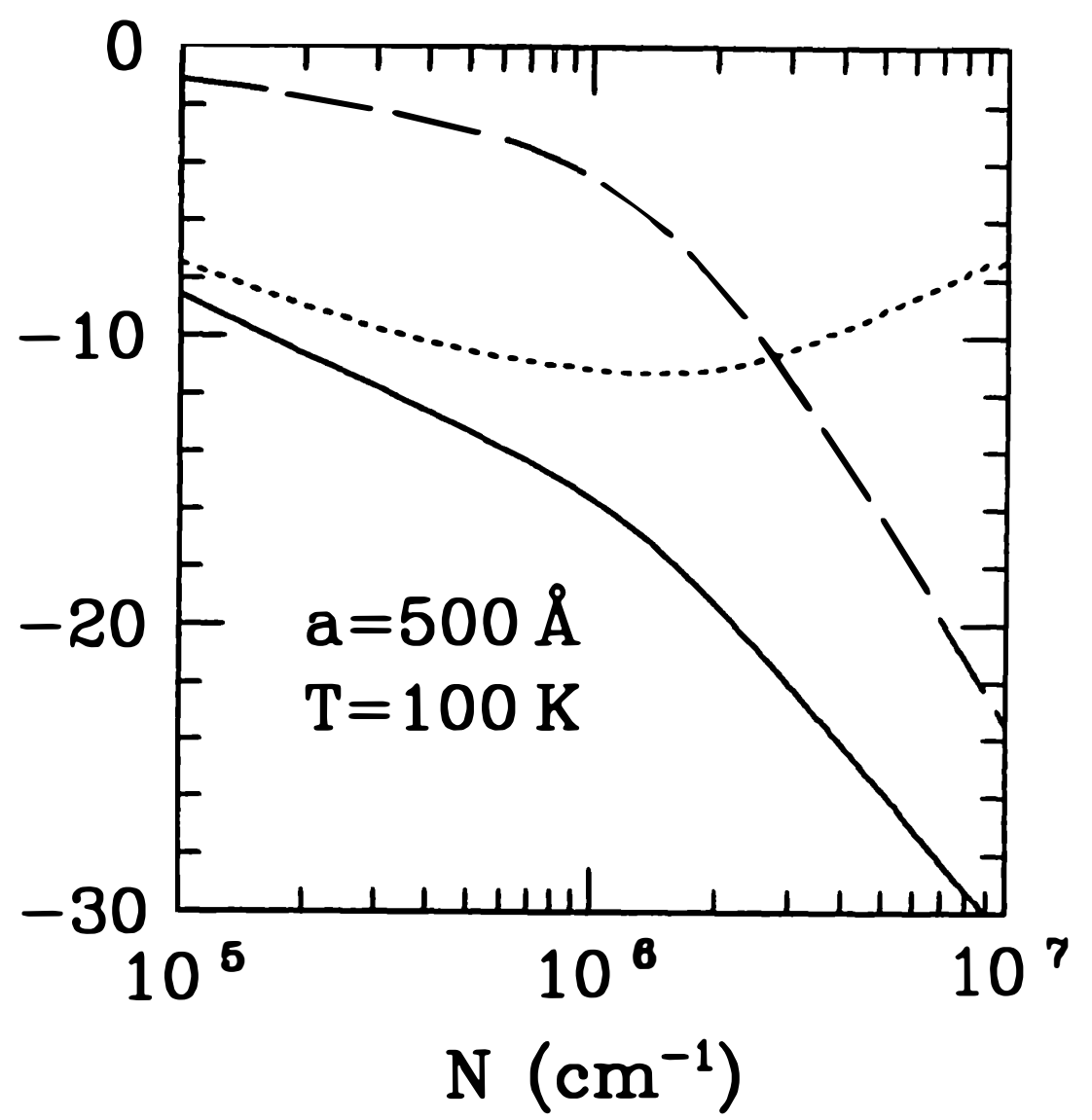

Figure 2. The sx (dashed line) and Ch (dotted line) contributions to the total band-gap renormalization (solid line) for $a=500 \AA$ wide quantum wire at $T=100 \mathrm{~K}$. 
the plasmon-pole approximation the static dielectric function is expressed as[3]

$$
\varepsilon(q)=1+\frac{\omega_{p}^{2}}{\frac{N q^{2}}{\mu \kappa}+\left(\frac{q^{2}}{2 \mu}\right)},
$$

where the plasmon frequency for the Q1D system is $\omega_{p}^{2}=(N / \mu) V(q)$, and the screening parameter is $\kappa=\sum_{i} \partial N / \partial \mu_{i}^{0}$. Here $\mu^{-1}=m_{e}^{-1}+m_{h}^{-1}$ is the reduced mass. This is essentially the approach taken by Benner and Haug[3], where they use a parabolic confinement potential. The plasmonpole approximation consists of ignoring the weight of single-particle excitations and assuming that all the weight of the dynamic susceptibility $\chi_{0}(q, \omega)$ is at an effective plasmon energy $\omega_{p}$. It correctly describes the static and long wavelength limits of the full RPA expression. Most BGR calculations [c.f. Refs. 8,9,10, and 11] are performed in the plasmon-pole approximation and its justification is rarely addressed. Das Sarma et al.[16] have found significant deviations of the plasmon-pole approximation from the full RPA results in quantum wells. The qualitative similarity of dotted and solid curves in Fig. 1 demonstrates the applicability of plasmon-pole approximation in Q1D systems in contrast to Q2D systems as found by Das Sarma et al.[16] We have calculated the $\varepsilon(q)$ within the temperature dependent RPA and the plasmon-pole approximation and found that they are quite similar. The RPA calculation is performed using Eq. (2) at a finite temperature, since the thermal electron and hole distribution functions $f_{i}(k)$ are used. The temperature dependence of $\varepsilon(q)$ in the plasmon-pole approximation comes from the screening parameter $\kappa$. Our calculations indicate that the plasmon-pole approximation becomes better for large $T$.

In Fig. 3 we show the temperature dependence of the band-gap renormalization $\Delta E_{g}$ in the Q1D electron system. The solid lines indicate BGR for a system at $N=10^{5} \mathrm{~cm}^{-1}$ with $a=100 \AA$ (lower curve) and $a=500 \AA$ (upper curve). The dotted lines are for $N=10^{6} \mathrm{~cm}^{-1}$ with $a=100 \AA$ (lower curve) and $a=500 \AA$ (upper curve). The results shown in Fig. 3 were calculated using the full RPA dielectric function at finite temperature, but we found that plasmon-pole approximation also works quite well. $\mathrm{Hu}$ and Das Sarma[4] have also investigated the temperature dependence of the BGR within the leading-order dynamical screening approximation ( $G W$ approximation). Our statically screened approximation yields qualitatively similar results suggesting dynamical screening is not significant in the range of plasma densities of experimental interest.

We have evaluated the renormalized chemical potential of the electronhole plasma including the exchange-correlation contribution as set out in the previous section. The total chemical potential $\mu_{T}$ of the Q1D electronhole system with well width $a=600 \AA$, for $k_{B} T=8$ and $16 \mathrm{meV}$ as 


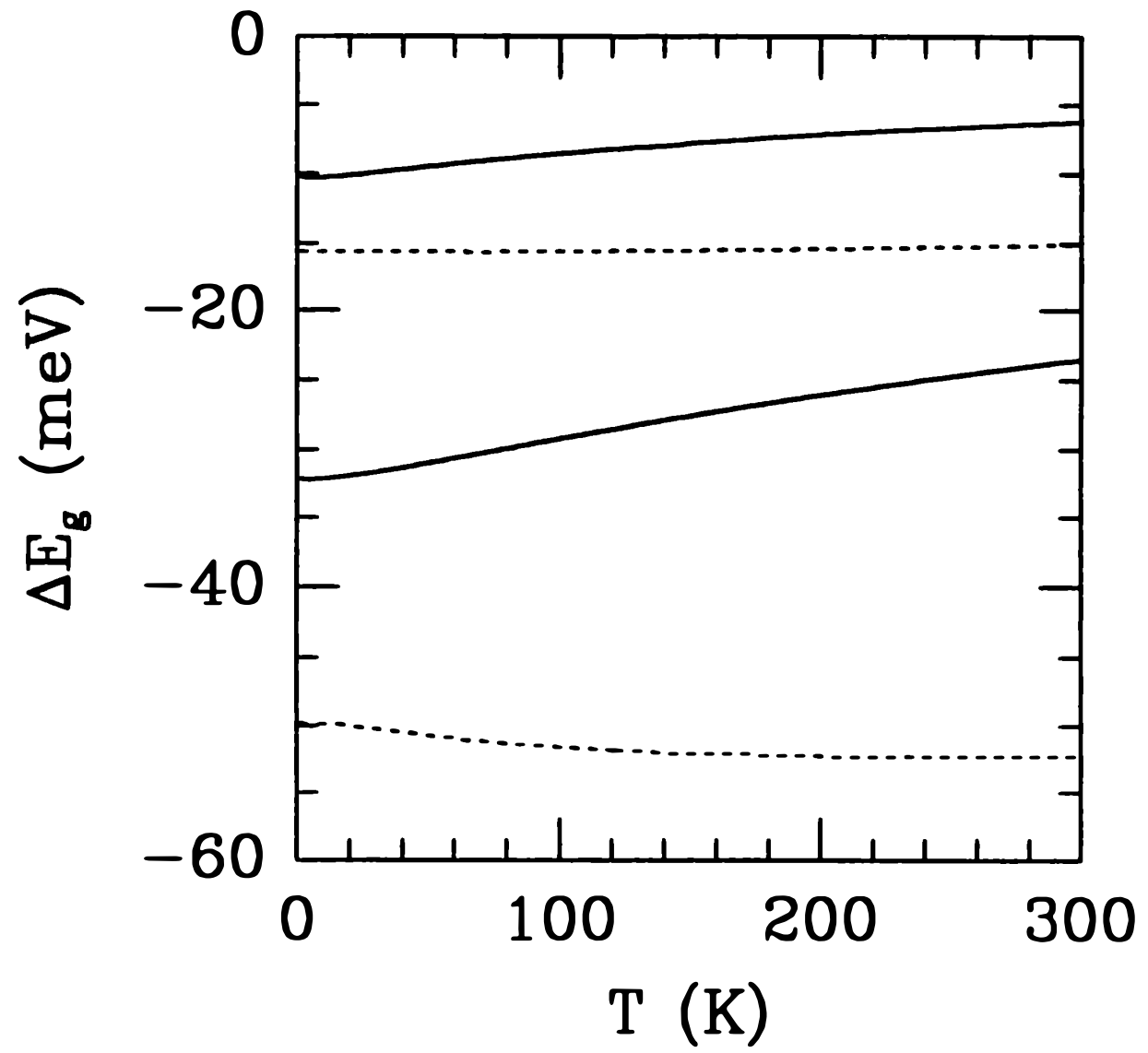

Figure 3. The temperature dependence of the band-gap renormalization for $N=10^{5}$ (solid lines) and $N=10^{6} \mathrm{~cm}^{-1}$ (dotted lines). The upper and lower curves are for $a=500$ and $a=100 \AA$ wide quantum-well wires.

a function of the plasma density is in qualitative agreement[5] with the experimental results of Cingolani et al.[13] obtained for similar parameters [c.f. Fig. 4 of Ref.13]. We found that there is a quantitative disagreement with the experiment especially for large densities, which may be attributed to the subband effects. Our calculations provide some indication about the rigid bandshift assumption. Using Eq.(6), we have calculated the exchange-correlation part of the chemical potential with both $\Sigma(k=0)$ and $\Sigma\left(k=k_{F}\right)$ and found no notable difference which suggests that the bandshifts occur rigidly. Figure 4 exhibits the band-gap renormalization at the band-edge $(k=0)$ and at $k_{F}$, for quantum-wire widths $a=500 \AA$ (solid line) and $a=1000 \AA$ (dashed line).

There seems to be a discrepancy in the band-gap renormalization between theory and experiment at high densities for Q2D structures. The origin of this general disagreement is not well understood. Several attempts to improve the theory, particularly the multisubband population case, did not change the qualitative behavior of the BGR. To explore the existence of similar behavior it would be interesting to perform experiments in Q1D structures at higher densities.

We now discuss the effects beyond the RPA, the local-field corrections to the BGR in quantum wires. Writing the dielectric function as $\varepsilon(q)=$ 


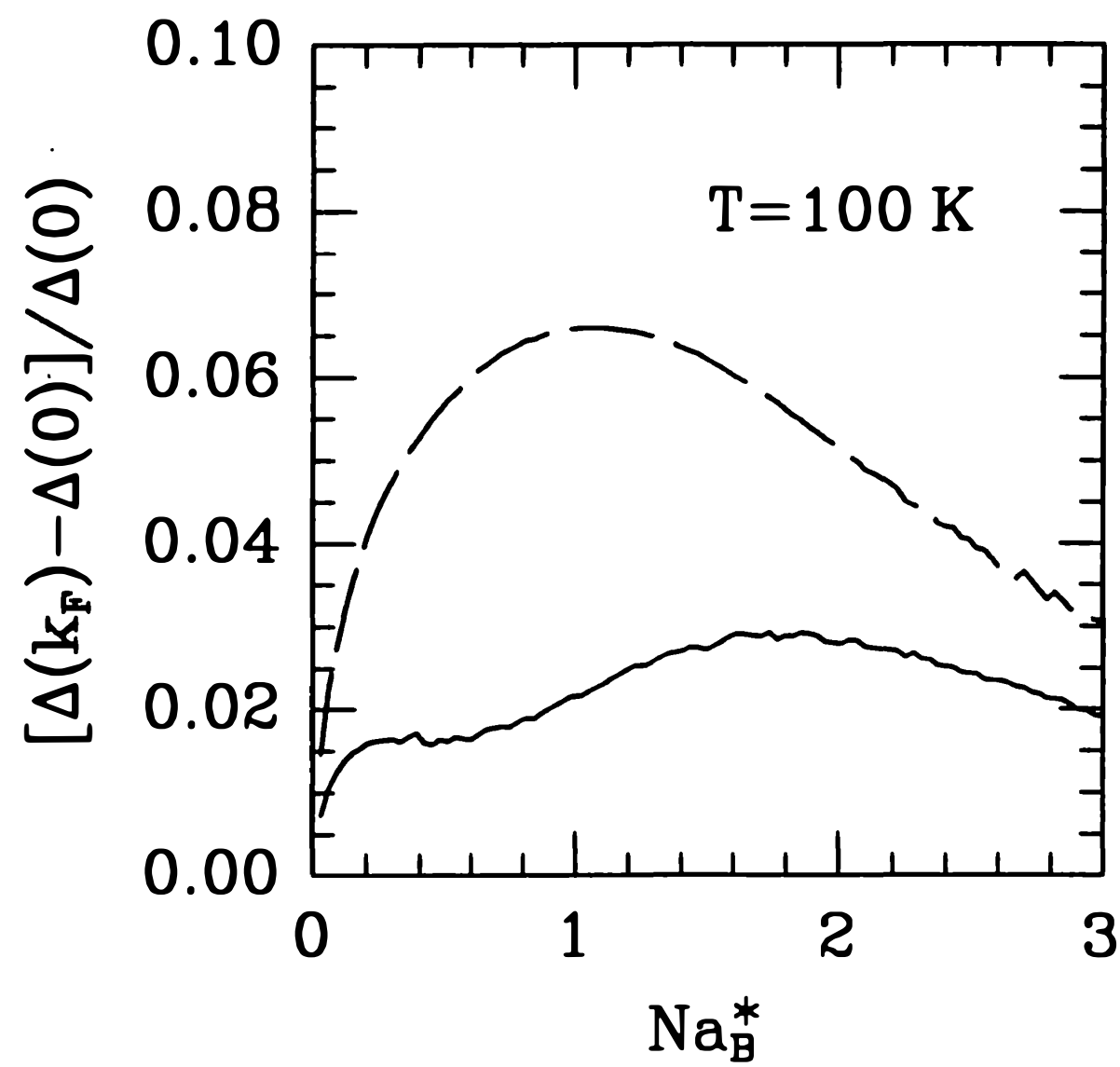

Figure 4. The total self-energy calculated at $k=k_{F}$ and at the band-edge $(k=0)$ as a function of the plasma density (we scale the density using the effective Bohr radius $a_{B}^{*}=\epsilon_{0} \hbar^{2} / \mu e^{2}$ in terms of the reduced mass $\mu$ and dielectric constant $\left.\epsilon_{0}\right)$. The solid and dotted curves indicate $a=500 \AA$ and $a=1000 \AA$ wide quantum-wires.

$1-V(q) \operatorname{II}(q)[1-G(q)]$, where $G(q)$ is the static local-field factor and $\operatorname{II}(q)$ is the static polarizability, we may account for the vertex corrections to $\operatorname{II}(q)$ in the mean-field sense. Recently, Schuster, Ell and Haug[17] considered finite-temperature vertex corrections in the form of second-order exchange contribution to the self-energy in $2 \mathrm{D}$ and $3 \mathrm{D}$ electron-hole plasmas. We use the equivalent of Hubbard approximation for $G(q)$ in one-dimension to obtain[18]

$$
G(q)=\frac{1}{2} \frac{V\left(\sqrt{q^{2}+k_{F}^{2}}\right)}{V(q)} .
$$

The physical nature of the Hubbard approximation is such that it takes exchange into account and corresponds to using the Pauli hole in the calculation of the local field correction between the particles of the same kind. Coulomb correlations are omitted. In this simple form, the static local-field factor $G(q)$ is temperature independent. Fig. 5 shows the BGR for quantum wires of various lateral widths at $T=100 \mathrm{~K}$ with and without the local field corrections. The solid curves are calculated with the local field correction whereas the dotted curves give the RPA $(G(q)=0)$. We observe that within the simple Hubbard approximation to $G(q)$, the BGR 


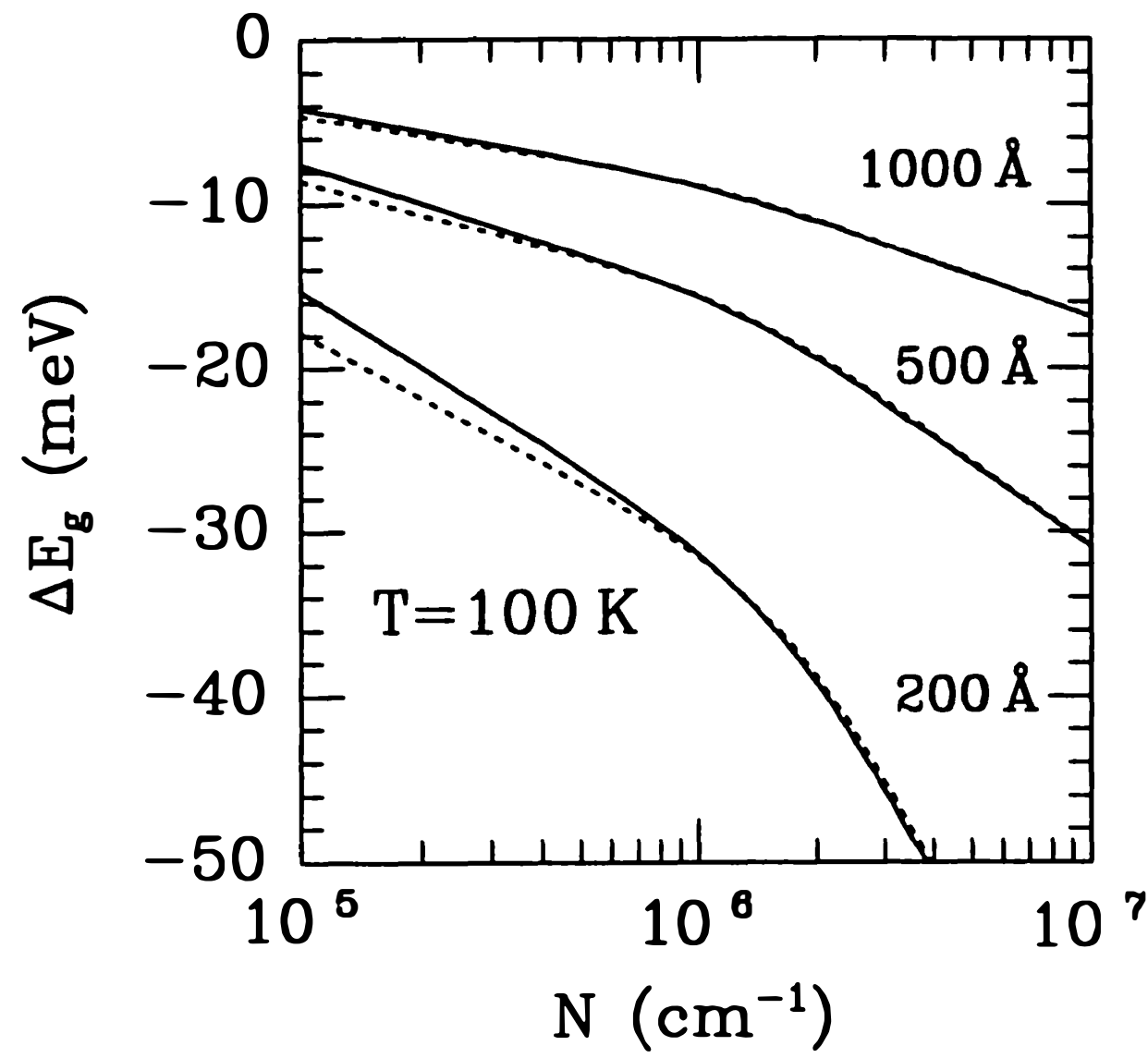

Figure 5. Effects of local-field corrections on the band-gap renormalization as a function of the plasma density at $T=100 \mathrm{~K}$. Dotted and solid lines are calculated with and without local-field corrections, respectively.

deviates from the RPA result as the quantum-well width decreases. The difference in BGR with and without $G(q)$ is more appreciable for lower densities. We argue that in general RPA is a good approximation for high densities, but requires modifications for low densities. In order to assess a reliable measure of corrections beyond RPA, better approximations to the local field factor $G(q)$ are needed. Returning to the issue of discrepancy in the calculated $\Delta E_{g}$ and measurements for high densities in Q2D systems, it seems unlikely that improvements of RPA could yield satisfactory agreement. Elaborate calculations of Schuster et al.[17] give an indication in this direction. Clearly, more experimental measurements of the type reported by Cingolani et al.[13,14] covering a wide range of plasma densities are necessary to resolve these questions.

As pointed out earlier, the band-gap renormalization accounts for the optical nonlinearities in the photoexcited semiconductor structures. The theoretical description of these phenomena in Q2D and 3D systems has been reviewed by Haug and Schmitt-Rink.[19] It would be interesting to examine the nonlinear optical properties of Q1D electron-hole systems using a similar approach. It is also possible to investigate the effects of electron-phonon interaction on the band-gap renormalization. Electron-phonon contribution to the BGR in Q1D systems were calculated by Güven and Tanatar[20] 
within perturbation theory and variational approaches. Recently, Dan and Bechstedt[21] treated the carrier-carrier and carrier-phonon interactions on an equal footing to calculate the phonon effects in Q1D systems. These calculations indicate the importance of the contribution of phonons to the BGR and their density dependence. The BGR due to carrier-carrier interactions increases with plasma density, whereas the carrier-phonon interactions tend to decrease $\Delta E_{g}$ at high densities.[20,21]

For the Q1D electron system we have used the model developed by $\mathrm{Hu}$ and Das Sarma[4] which introduces an additional confinement to an infinite square-well. There are various other models of the quantum-well wire structures using parabolic confining potentials, geometrical reduction of dimensionality. The general trends obtained here for the plasma density and temperature dependence should be valid irrespective of the details of the model chosen.

Although we have carried out our numerical calculations for the material parameters of GaAs, the same formalism may be applied to other semiconductor structures such as In As, GaSb, AlAs, etc. It would be desirable to have experimental results of the BGR for different Q1D semiconductor materials to compare with theoretical calculations. In $2 \mathrm{D}$ and $3 \mathrm{D}$, a somewhat universal dependence of $\Delta E_{g}$ on plasma density is established largely independent of the band structure details. Whether a similar general behavior exists in Q1D electron-hole systems would be settled as more photoluminescence experiments become available.

\section{Summary}

We have found that the static plasmon-pole approximation to the dielectric function yields very close results to the full RPA expression. The temperature dependence of the BGR is weak for densities $N \sim 10^{6} \mathrm{~cm}^{-1}$. Localfield corrections employed within the Hubbard approximation decreases the BGR at low densities especially when the lateral width of the quantum wire is small. The calculated renormalized chemical potential of the electron-hole plasma qualitatively differs from the measurement which may be attributed to the subband effects.

Extension of our calculations to cases where more than one subband is populated would be interesting for comparison with future experiments. It was recently found by Ryan and Reinecke[22] that in Q2D systems the intersubband interactions make significant contribution to the band-gap renormalization. More experimental results are needed in quantum wires to discuss fully the various aspects of BGR. Given the importance of the Coulomb interaction, it would be useful to analyze the experimental results with more refined line-shape models. 
We gratefully acknowledge the partial support of this work by the Scientific and Technical Research Council of Turkey (TUBITAK) under Grant No. TBAG-AY/77, and fruitful discussions with Professors R. Cingolani, E. Kapon, and C. Sotomayor-Torres.

\section{References}

1. Schmitt-Rink S., Chemla D. S., and Miller D. A. B., (1989) Linear and nonlinear optical properties of semiconductor quantum wells, Adv. Phys. 38, 89; Cingolani R. and Ploog K., (1991) Frequency and density dependent radiative recombination processes in III-V semiconductor quantum wells and superlattices, Adv. Phys. 40, 535.

2. Plaut A. S., Lage H., Grambow P., Heitmann D., von Klitzing K., and Ploog K., (1991) Direct magneto-optical observation of a quantum confined one-dimensional electron gas, Phys. Rev. Lett. 67, 1642; Goni A. R., Wiener J. S., Calleja J. M., Dennis B. S., Pfeiffer L. N., and West K. W., (1991) One-dimensional plasmon dispersion and dispersionless intersubband excitations in GaAs quantum wires, Phys. Rev. Lett. 67, 3298.

3. Benner S. and Haug H., (1991) Plasma-density dependence of the optical spectra for quasi-one-dimensional quantum well wires, Europhys. Lett. 16, 579; (1993) Influence of external electric and magnetic fields on the excitonic absorption spectra of quantum-well wires, Phys. Rev. B 47, 15570.

4. Hu B. Y.-K. and Das Sarma S., (1992) Many-body properties of a quasi-onedimensional semiconductor quantum wire, Phys. Rev. Lett. 68, 1750; (1993) Manybody exchange-correlation effects in the lowest subband of semiconductor quantum wires, Phys. Rev. B 48, 5469.

5. Tanatar B., (1996) Band-gap renormalization in quasi-one-dimensional electron-hole systems, J. Phys. Condens. Matter 8, 5997.

6. Wang K. H., Bayer M., Forchel A., Ils P., Benner S., Haug H., Pagnod-Rossiaux Ph., and Goldstein L., (1996) Subband renormalization in dense electron-hole plasmas in $\mathrm{In}_{0.53} \mathrm{Ga}_{0.47} \mathrm{As} / \mathrm{InP}$ quantum wires, Phys. Rev. B 53,10 505.

7. Grundman M., Christen J., Joschko M., Bimberg D., and Kapon E., (1995) Bandgap renormalization in quantum wires, in D. J. Lockwood (ed.), The Physics of Semiconductors, World Scientific, Singapore, p. 1675.

8. Haug H. and Schmitt-Rink S., (1984) Electron theory of the optical properties of laser-excited semiconductors, Prog. Quantum Electron. 9, 3.

9. Schmitt-Rink S., Ell C., Koch S. W., Schmidt H. E., and Haug H., (1984) Subbandlevel renormalization and absorptive optical bistability in semiconductor multiple quantum well structures, Solid State Commun. 52, 123; Haug H. and Koch S. W., (1989) Semiconductor laser theory with many-body effects, Phys. Rev. A 39, 1887.

10. Ell C., Blank R., Benner S., and Haug H., (1989) Simplified calculations of the optical spectra of two- and three-dimensional laser-excited semiconductors, J. Opt. Soc. Am. B 6, 2006.

11. Ell C., Haug H., and Koch S. W., (1989) Many-body effects in gain and refractiveindex spectra of bulk and quantum-well semiconductor lasers, Opt. Lett. 14, 356.

12. Tränkle G., Lach E., Forchel A., Scholz F., Ell C., Haug H., Weimann G., Griffiths G., Kroemer H., and Subbanna S., (1987) General relation between band-gap renormalization and carrier density in two-dimensional electron-hole plasmas, Phys. Rev. B 36, 6712; Tränkle G., Leier H., Forchel A., Haug H., Ell C., and Weimann G., (1987) Dimensionality dependence of the band-gap renormalization in two- and three-dimensional electron-hole plasmas in GaAs, Phys. Rev. Lett. 58, 419; Bongiovanni G. and Staehli J. L., (1989) Properties of the electron-hole plasma in GaAs- $(\mathrm{Ga}, \mathrm{Al})$ As quantum wells: the influence of the finite well width, Phys. Rev. 
B 39, 8359; Weber C., Klingshirn C., Chemla D. S., Miller D. A. B., Cunningham J. E., and Ell C., (1988) Gain measurements and band-gap renormalization in $\mathrm{GaAs} / \mathrm{Al}_{x} \mathrm{Ga}_{1-x} \mathrm{As}$ multiple-quantum-well structures, Phys. Rev. B 38, 12 748; Kulakovskii V. D., Lach E., Forchel A., and Grützmacher D., (1989) Band-gap renormalization and band-filling effects in a homogeneous electron-hole plasma in $\mathrm{In}_{0.53} \mathrm{Ga}_{0.47} \mathrm{As} / \mathrm{InP}$ single quantum wells, Phys. Rev. B 40, 8087.

13. Cingolani R., Rinaldi R., Ferrara M., La Rocca G. C., Lage H., Heitmann D., Ploog K., and Kalt H., (1983) Band-gap renormalization in quantum wires, Phys. Rev. B 48, 14331.

14. Cingolani R., Lage H., Tapfer L., Kalt H., Heitmann D., and Ploog K., (1991) Quantum confined one-dimensional electron-hole plasma in semiconductor quantum wires, Phys. Rev. Lett. 67, 891.

15. Güven K. and Tanatar B., (1996) Simplified calculations of band-gap renormalization in quantum-wells, Superlatt. Microstruct. 20, 81; and references therein.

16. Das Sarma S., Jalabert R., and Eric Yang S.-R., (1989) Band-gap renormalization in quasi-two-dimensional systems induced by many-body electron-electron and electron-phonon interactions, Phys. Rev. B 39, 5516; (1990) Band-gap renormalization in semiconductor quantum wells, ibid. 41, 8288.

17. Schuster S., Ell C., and Haug H., (1992) Vertex correction to the single-particle energy renormalization in three- and two-dimensional electron-hole plasmas, Phys. Rev. B 46, 16167.

18. Gold A. and Ghazali A., (1990) Analytical results for semiconductor quantum-well wire: plasmons, shallow impurity states, and mobility, Phys. Rev. B 41, 7626.

19. Haug H. and Schmitt-Rink S., (1985) Basic mechanisms of the optical nonlinearities of semiconductors near the band edge, J. Opt. Soc. Am. B 2, 1135.

20. Güven K. and Tanatar B., (1995) Phonon renormalization effects in photoexcited quantum wires, Phys. Rev. B 51, 1784; (1996) Variational approach for phonon renormalization effects in photoexcited quantum wires and quantum wells, phys. status solidi b 197,369 .

21. Dan N. T. and Bechstedt F., (1996) Optical phonon effects in quasi-one-dimensional semiconductor quantum wires: band-gap renormalization, Physica B 219\&220, 47.

22. Ryan J. C. and Reinecke T. L., (1993) Band gap renormalization in semiconductor quantum wells, Superlatt. Microstruct. 13, 177; (1993) Band-gap renormalization of optically excited semiconductor quantum wells, Phys. Rev. B 47, 9615. 